\title{
Effect of Pacing Site on the Atrial Electrogram at Target Sites for Slow Pathway Ablation in Patients with Atrioventricular Nodal Reentrant Tachycardia
}

\author{
JOHN HUMMEL, S. ADAM STRICKBERGER, STEVEN KALBFLEISCH, \\ BRIAN WILLIAMSON, K. CHING MAN, VICKEN VORPERIAN, \\ FRED MORADY, and JONATHAN LANGBERG \\ From the Division of Cardiology, University of Michigan Medical Center, Ann Arbor, Michigan
}

\begin{abstract}
HUMMEL, J., ET AL.: Effect of Pacing Site on the Atrial Electrogram at Target Sites for Slow Pathway Ablation in Patients with Atrioventricular Nodal Reentrant Tachycardia. Atrial electrograms recorded from target sites during radiofrequency catheter ablation of the slow atrioventricular ( $A V$ ) nodal pathway are often fractionated and may be associated with a late, high frequency component (the slow pathway potential). The purpose of the current study was to assess the effects of slow pathway ablation on the morphology of the atrial electrogram and to determine whether target site electrograms display direction dependent changes in morphology during atrial pacing maneuvers. Twenty-six patients with typical AV nodal reentry had electrograms recorded from target sites before and after successful ablation of the slow AV nodal pathway and during pacing from the high right atrium and distal coronary sinus at cycle lengths of 500 and $300 \mathrm{msec}$. There was no significant change in the duration or degree of fractionation of the atrial electrogram as the result of slow pathway ablation. In contrast, the duration and degree of fractionation were less when pacing from the coronary sinus compared with sinus rhythms or right atrial pacing. Pacing rate did not affect electrogram morphology. These data suggest that the morphology of the slow pathway target site electrogram is dependent on the direction of atrial activation and that the "slow pathway potential" does not represent activation of an anatomically discrete pathway. (PACE, Vol. 17, April 1994, Part I)
\end{abstract}

radiofrequency catheter ablation, supraventricular tachycardia, anisotropic conduction

\section{Introduction}

Multicomponent atrial electrograms are useful as a guide for radiofrequency ablation of the slow pathway in patients with typical atrioventricular nodal reentrant tachycardia (AVNRT). These target site electrograms have been analyzed in an attempt to define the functional and anatomical

\footnotetext{
Address for reprints: John Hummel, M.D., University of Michigan Medical Center, Division of Cardiology, 1500 East Medical Center Drive, Ann Arbor, MI 48109-0022. Fax: (313) 763-7390. Received June 18, 1993; revision October 27, 1993; accepted December 6, 1993.
}

properties of the slow pathway. ${ }^{1-3}$ A prior study examined the effect of rate, but none have examined the effect of pacing site on electrogram morphology. Therefore, the purpose of this study was to determine whether target site electrograms display direction dependent changes in morphology during atrial pacing maneuvers.

\section{Methods}

\section{Characteristics of Subjects}

The subjects of this study were 26 patients with typical AVNRT who underwent successful radiofrequency ablation of the slow pathway. Pa- 
tients with atypical AVNRT and patients in whom an electrode catheter could not be introduced into the coronary sinus were excluded from the study. There were 10 men and 16 women with a mean age of $44 \pm 19$ years. Twenty-five patients did not have structural heart disease. One patient had longstanding hypertension and left ventricular hypertrophy.

\section{Electrophysiological Testing}

Electrophysiological testing was performed in the fasting state after informed consent was obtained. Each patient underwent a baseline electrophysiological test at least five half-lives after discontinuation of antiarrhythmic drugs. Three quadripolar electrode catheters were inserted into a femoral vein and positioned in the right ventricular apex, His-bundle region, and the high right atrium. Leads $\mathrm{V}_{1}, \mathrm{I}$, II, and III and the intracardiac electrograms were displayed on an oscilloscope and recorded at a paper speed of 25 to $100 \mathrm{~mm} / \mathrm{sec}$ on a Mingograf 7 recorder (Siemens-Elema, Solna, Sweden). Intracardiac electrograms were recorded at a band-pass of 50 to $500 \mathrm{~Hz}$. Pacing was performed using a programmable stimulator (Bloom Associates, Ltd., Reading, PA, USA). Pacing stimuli had a duration of $2 \mathrm{msec}$ and a current strength twice the late diastolic threshold.

Atrial and ventricular overdrive pacing and programmed stimulation were performed to define the conduction properties and refractory period of the AV node and to induce supraventricular tachycardia. The presence of AVNRT was established using standard criteria. ${ }^{1-3}$

\section{Ablation and Study Protocol}

After the diagnostic portion of the electrophysiological test, the right ventricular quadripolar catheter was placed in the coronary sinus. A deflectable 7-French bipolar or quadripolar ablation catheter with 2-mm interelectrode spacing and a 4-mm platinum tip electrode was used to map the posteromedial portion of the tricuspid annulus. Ablation sites were selected based on the morphology of the local atrial electrogram. Target site electrograms were sought that demonstrated an $\mathrm{A} / \mathrm{V}$ ratio of $<0.5$ and a multicomponent atrial electrogram. All target site electrograms were recorded at a gain of $80 \mathrm{~mm} / \mathrm{mV}$ and a paper speed of $100 \mathrm{~mm} / \mathrm{sec}$. Radiofrequency energy at a frequency of $500 \mathrm{kHz}$ was delivered as a continuous, unmodulated sine wave (EP Technologies, Sunnyvale, CA, USA) between the distal electrode of the ablation catheter and a patch electrode on the back (Valley Lab Inc., Boulder, CO, USA). Radiofrequency current was usually delivered at a power of 30 to 35 watts for 15 to 30 seconds. Radiofrequency energy was applied until there was elimination of slow pathway function, defined as the absence of inducibility of AVNRT. This was usually, but not invariably, associated with the loss of dual AV nodal physiology as well. The effects of ablation on target site atrial electrogram morphology were assessed by comparing tracings recorded before and immediately after successful ablation. In order to define the effects of activation sequence on slow pathway target site electrogram morphology, overdrive pacing was performed for 5 to 10 seconds from the high right atrium and from the distal coronary sinus at cycle lengths of 500 and 300 msec while recording from each successful site.

\section{Analysis of Data}

All target site electrograms were acquired under similar conditions and analyzed using the identical criteria. The duration of the bipolar target site atrial electrogram was defined as the time from the initial to the terminal atrial deflection, including the high frequency terminal component-the "slow pathway potential." The degree of fractionation of the atrial signal was quantitated by counting the number of local peaks or components, with a peak being defined as having a minimum amplitude of $0.025 \mathrm{mV}$ and a minimum duration of 10 msec. Electrograms were analyzed and measured in a blinded fashion by two observers and compared to assess reproducibility. Statistical comparisons were performed using Student's $t$-test. A P value $<0.05$ was considered significant.

\section{Results}

\section{Atrial Electrogram Duration}

The mean target site atrial electrogram duration during sinus rhythm demonstrated no significant change from baseline when measured after ablation (Fig. 1). The mean atrial electrogram dura- 

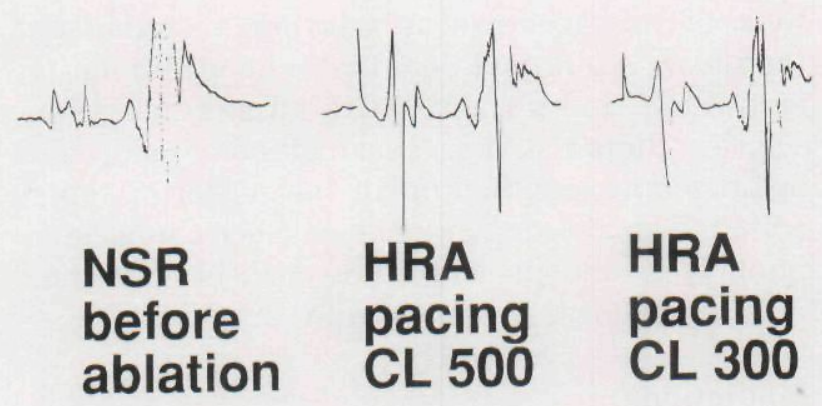

HRA pacing

HRA CL 500 pacing CL 300

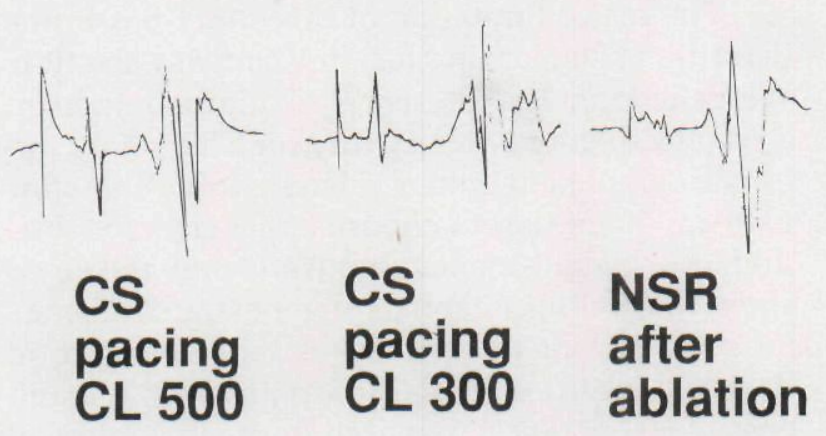

Figure 1. Bipolar recordings from a single target site before and after successful catheter ablation of the slow $A V$ nodal pathway, as well as during pacing from the right atrium and coronary sinus. Note the persistence of a late, high frequency spike (a "slow pathway" potential) after ablation. In contrast, pacing from the coronary sinus resulted in a decrease in the duration and fractionation of the atrial electrogram. $C L=$ cycle length; $C S=$ coronary sinus; HRA = high right atrium; $N S R=$ normal sinus rhythm.

tion was significantly shorter when pacing from the coronary sinus as compared to the high right atrium at a cycle length of $500 \mathrm{msec}(62.5 \pm 20 \mathrm{vs}$ $71.3 \pm 16.5 \mathrm{msec}, \mathrm{P}<0.05)$, and also at a cycle length of $300 \mathrm{msec}(60.4 \pm 20$ vs $70.2 \pm 18 \mathrm{msec}$, $\mathrm{P}<0.05)$. The mean atrial electrogram durations at pacing cycle lengths of 500 and $300 \mathrm{msec}$ did not differ significantly either during pacing of the high right atrium or coronary sinus (Fig. 2).

\section{Atrial Electrogram Components}

During sinus rhythm, there was no significant difference in the number of peaks before ablation as compared to after ablation (Fig. 1). There was no statistical difference in the number of peaks in the target site atrial electrograms during right atrial pacing compared with sinus rhythm (Fig. 3). How-

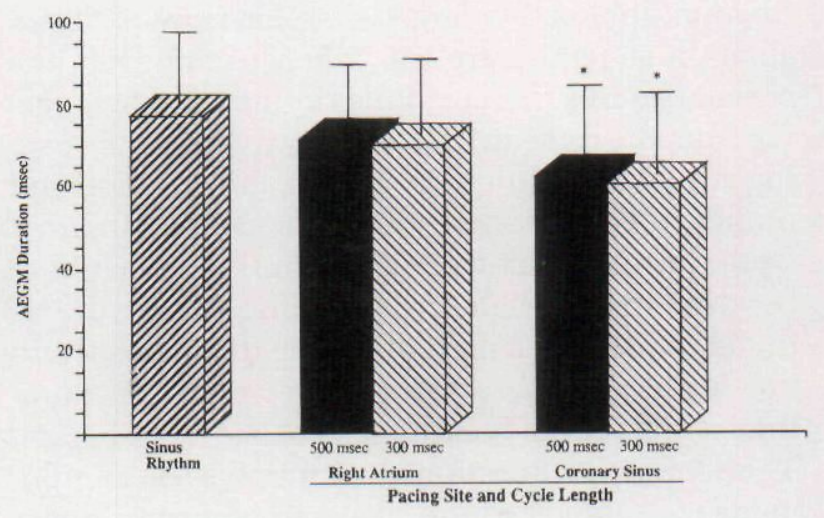

Figure 2. The duration of the target site atrial electrogram in sinus rhythm compared to that recorded during right atrial and coronary sinus pacing. AEGM = atrial electrogram; * $=P<0.05$ versus sinus rhythm.

ever, during coronary sinus pacing, there were significantly fewer peaks than during high right atrial pacing (Fig. 3). Changes in the pacing cycle length did not affect the number of peaks present in the atrial electrogram.

\section{Discussion}

\section{Main Findings}

This study demonstrates that successful ablation of the slow AV nodal pathway does not affect the duration or degree of fractionation of the target site atrial electrogram. In contrast, the duration and number of components of the atrial elec-

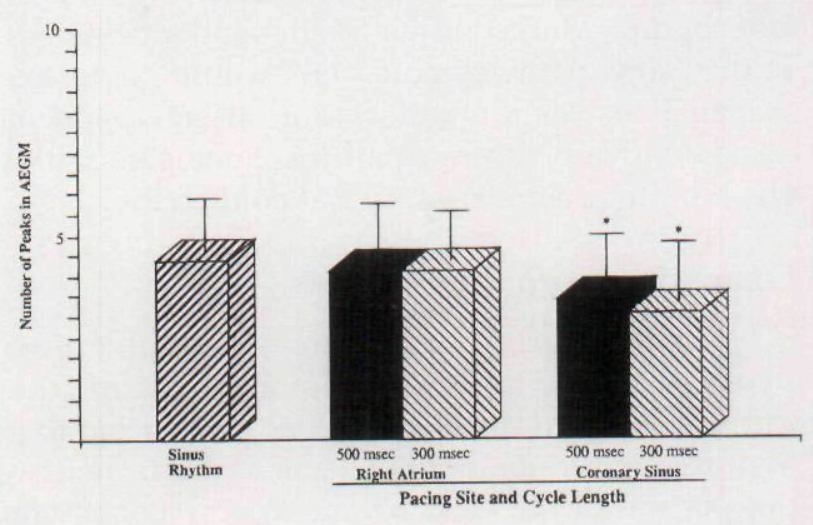

Figure 3. Degree of fractionation as manifest by the number of peaks in the atrial electrogram versus pacing site and cycle length. Abbreviations as in Figure 2. 
trogram at effective target sites for slow pathway ablation generally are less when pacing from the coronary sinus than in sinus rhythm or when pacing from the right atrium. With coronary sinus pacing at a cycle length of $500 \mathrm{msec}$, the duration and number of components of the local electrogram were noted to decrease significantly compared to baseline and to right atrial pacing. However, reducing the cycle length of pacing to $300 \mathrm{msec}$ did not further decrease the atrial electrogram duration or number of components of the electrogram. Therefore, the duration and number of components of the target site atrial electrogram are dependent on the direction of activation, but not on pacing rate.

\section{Mechanistic Implications}

In nonuniformly anisotropic tissue, axial resistivity is considerably higher than resistivity in parallel to the axis of fiber orientation. ${ }^{4-6}$ Activation that proceeds transverse to fiber orientation is relatively slow and characteristically produces a prolonged, fractionated extracellular electrogram. ${ }^{7}$ The atrial myocardium adjacent to the ostium of the coronary sinus that serves as the posterior input into the AV node is associated with fractionated electrograms in animals. ${ }^{8,9}$ The degree of anisotropy in this region may determine whether there is sufficient slowing of conduction to allow sustained AV nodal reentry. The direction dependent changes in electrogram duration and fractionation observed in this study are consistent with the hypothesis that slow pathway function is mediated by a highly anisotropic region of the posterior septum. Marked direction dependent changes in the "slow pathway potential" would not be expected if it was a manifestation of activation in an anatomically discrete pathway analogous to the His bundle or an accessory AV connection.

\section{Comparison with Prior Studies}

The local atrial electrograms at effective sites in our series had multiple components within the initial portion of the electrogram, in addition to a high frequency component separate from the initial portion of the electrogram. With a change in pacing direction, many of these high frequency terminal components completely or partially disappeared (Fig. 1). These observations concur with those of Haissaguerre et al. ${ }^{1}$ who demonstrated that the separate terminal potentials changed polarity, amplitude, and duration with atrial stimulation. These authors also suggested that there is slow and heterogenous conduction in the posterior septal region. The site dependency of electrogram morphology in the present study also is consistent with a role for tissue anisotropy in AVNRT.

\section{Limitations}

The major limitation of this study lies in the definition of target sites for slow pathway ablation. We relied on the presence of multicomponent atrial electrograms along with a high frequency terminal component (often referred to as the slow pathway potential) to choose target sites for ablation. No pacing maneuvers were undertaken to show that these potentials demonstrated characteristics previously defined as representative of the atrial insertion of the slow pathway. ${ }^{10} \mathrm{~A}$ bandpass of 50 to $500 \mathrm{~Hz}$ was used during recording of target site electrograms. We cannot rule out the possibility that some direction dependent changes were attenuated by filtering or partially due to the direction of the wavefront with respect to bipolar orientations. It does seem likely that these factors could account entirely for the marked changes in the duration of the target site electrogram after a change in atrial pacing site.

Pre- and postablation pacing maneuvers would have eliminated any question of local electrogram alteration by the application of radiofrequency energy to the area. This was not feasible due to time constraints; however, the local electrogram in sinus rhythm was not altered by ablation, making it unlikely that the response to pacing was altered.

\section{Conclusions}

Target site electrograms undergo changes in morphology and duration as the result of changes in pacing direction. These pacing maneuvers demonstrate direction dependent conduction properties of the tissue giving rise to these target electrograms. These properties are consistent with anisotropic conduction and suggest that the anterograde slow pathway in AVNRT may consist of anisotropic regions of perinodal atrium instead of an anatomically discrete pathway. 


\section{ELECTROGRAM MORPHOLOGY}

\section{References}

1. Haissaguerre M, Gaita F, Fischer B, et al. Elimination of atrioventricular nodal reentrant tachycardia using discrete slow potentials to guide application of radiofrequency energy. Circulation 1992; 85:2162-2175.

2. Kay GN, Epstein AE, Dailey SM, et al. Selective radiofrequency ablation of the slow pathway for the treatment of atrioventricular nodal reentrant tachycardia. Circulation 1992; 85:1675-1688.

3. Jazayeri MR, Hempe SL, Sra JS, et al. Selective transcatheter ablation of the fast and slow pathways using radiofrequency energy in patients with atrioventricular nodal reentrant tachycardia. Circulation 1992; 85:1318-1328.

4. Spach MS, Miller WT, Geselowitz DB, et al. The discontinuous nature of propagation in normal canine cardiac muscle. Evidence for recurrent discontinuities of intracellular resistance that affect membrane currents. Circ Res 1981; 48:39-54.

5. Spach MS, Miller WT, Dolber PG, et al. The functional role of structural complexities in the propagation of depolarization in the atrium of the dog. Cardiac conduction disturbance due to discontinuities of effective atrial resistivity. Circ Res 1982; 50:175-191.
6. Lesh MD, Spear JF, Moore EN. Myocardial anisotropy: Basic electrophysiology and role in cardiac arrhythmias. In DP Zipes, J Jalife (eds.): Cardiac Electrophysiology. Philadelphia, PA, W.B. Sanders Co., 1990, pp. 364-376.

7. Spach MS, Dolber PC: Relating extracellular potentials and their derivatives to anisotropic propagation at a microscopic level in human cardiac muscle. Evidence for electrical uncoupling of side-toside fiber connections with increasing age. Circ Res 1986; 58:356-371.

8. Ilnuma H, Dreifus LS, Mazgalev T, et al. Role of the perinodal region in atrioventricular nodal reentry: Evidence in an isolated rabbit heart preparation. J Am Coll Cardiol 1983; 2:465-473.

9. Woods WT, Sherf L, James TN. Structure and function of specific regions in the canine atrioventricular node. Am J Physiol 1982; 243:H41-H50.

10. Jackman WM, Beckman KJ, McClelland JH, et al. Treatment of supraventricular tachycardia due to atrioventricular nodal reentry by radiofrequency catheter ablation of slow pathway conduction. N Engl J Med 1992; 327:313-318. 
This document is a scanned copy of a printed document. No warranty is given about the accuracy of the copy. Users should refer to the original published version of the material. 